\title{
Retraction Note to: relationships between PTEN gene mutations and prognosis in glioma: a meta-analysis
}

\author{
Wei-Zhong Xiao ${ }^{1}$ - Dong-Hua Han ${ }^{2}$ - Fei Wang ${ }^{3}$-Yong-Qian Wang ${ }^{4}$.

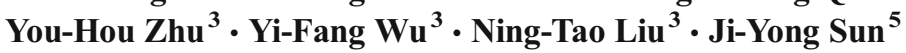

Published online: 17 August 2015

(C) International Society of Oncology and BioMarkers (ISOBM) 2015

Retraction to: Tumor Biol. (2014) 35:6687-6693

DOI 10.1007/s13277-014-1885-1

The Publisher and Editor retract this article in accordance with the recommendations of the Committee on Publication Ethics (COPE). After a thorough investigation we have strong reason to believe that the peer review process was compromised.

The online version of the original article can be found at http://dx.doi.org/ 10.1007/s13277-014-1885-1.

Fei Wang

wangfeiwf305@126.com

1 Department of Neurology, Shanghai Pudong Hospital, Fudan University, Shanghai 201399, People's Republic of China

2 Department of Neurosurgery, Shanghai Pudong Hospital, Fudan University, Shanghai 201399, People's Republic of China

3 Department of Neurosurgery, Tongji hospital, Tongji University, Xincun Road No. 389, Putuo District, Shanghai 200065, People's Republic of China

4 Department of Neurosurgery, Longhua Hospital Affiliated to Shanghai University of Traditional Chinese Medicine, Shanghai 201399, People's Republic of China

5 Department of Neurosurgery, Wuxi Second Hospital Affiliated to Nanjing Medical University, Wuxi 214002, People's Republic of China 